\title{
Individual differences in perceived pinch force and bite force
}

\author{
CLAYTON L. VAN DOREN \\ Case Western Reserve University, Cleveland, Ohio
}

\begin{abstract}
Six subjects made cross-modal matches of pinch force and bite force to an electrocutaneous stimulus. The electrocutaneous stimulus consisted of bursts of pulses, and the intensity of the stimulus was varied by changing the number of pulses per burst. All of the individual matching functions were fit well by power functions. The scaling constants and exponents of the power functions covaried systematically with the maximum pinch force for 5 of the 6 subjects. The relationship was consistent with the hypothesis that subjects perceive their physical maxima equally, in agreement with Borg's theory of relative perceived exertion. For both pinch force and bite force functions, the scale factors could be described by a single linear function of the exponents, suggesting that all of the matching functions converged at a single point with extreme values.
\end{abstract}

In an earlier paper (Van Doren, Riso, \& Milchus, 1991), we described a heuristic for implementing synthetic sensory feedback (i.e., the presentation of sensory information from an absent or damaged modality via an alternate modality) and presented initial results from our development of an electrocutaneous code for grasp or pinch force. Cross-modal matches between pinch force and the electrocutaneous stimulus showed marked intersubject differences that were closely related to subject strength-that is, their maximum pinch force or maximum voluntary contraction (MVC). Similar variations appeared in the bite force matches. Individual differences are a nuisance in the context of engineering a feedback system; however, in the context of basic psychophysics, such differences can provide a test for certain theories of perception.

Gunnar Borg, in particular, has proposed that different persons all perceive the same subjective level of exertion when they exercise at their respective physical maxima (Borg, 1961, 1962). If the psychophysical function for perceived exertion also has the same shape for all subjects, then exertion will be perceived equally at any specific fraction of their maxima (Marks, Borg, \& Ljunggren, 1983). The theory has been supported indirectly by using heart rate as a measure of relative exertion (Marks et al., 1983). At any given work load while pedaling a bicycle ergometer, subjects with a low heart rate tend to report low exertion values using Borg's relative perceived exertion (RPE) scale or magnitude estimation, whereas subjects with high heart rates report higher exertion values. Subjects with low heart rates operate, presumably, at lower fractions of their physical maxima than do subjects with high heart rates. Note, however, that Mihevic (1983) found no difference between the RPE ratings of groups of high- and low-fitness men and women

This research was supported by Grants NS 27958 and NS92355 from the National Institutes of Health. Correspondence should be addressed to C. L. Van Doren, MetroHealth Medical Center, H61 1, 2500 MetroHealth Drive, Cleveland, OH 44109-1998. (segregated on the basis of maximal oxygen uptake) at low to moderate work loads, even though heart rates were significantly different (see also Morgan \& Pollock, 1977; Nagle, Morgan, Hellickson, Serfass, \& Alexander, 1975; Patton, Morgan, \& Vogel, 1977).

A similar interpretation has been proposed for perceived muscular effort during static contractions (e.g., Roland \& Ladegaard-Pedersen, 1977): Two isometric contractions will be perceived as equal if the forces are equal proportions of their respective maxima. As in the case of dynamic exertion, a given force will feel more intense as the strength of the muscle decreases. Experiments have shown that perceived effort increases as the maximum force of a muscle decreases due to changes in length (Cafarelli \& Bigland-Ritchie, 1979), fatigue (McCloskey, Ebeling, \& Goodwin, 1974; Stevens \& Cain, 1970), or partial curarization (Roland \& Ladegaard-Pedersen, 1977). In a longitudinal study of perceived respiratory force, Redline, Gottfried, and Altose (1991) found similarly that inspiratory muscle training transiently decreased magnitude estimates of respiratory effort and that ratings varied inversely with maximal inspiratory pressure.

The theory of RPE can also be applied to our crossmodal matching data (pinch force or bite force to electrocutaneous intensity) in two steps. First, perceived force (or effort) is matched to a second continuum, $N$. In our particular case, $N$ represents the number of pulses in each burst of the electrocutaneous stimulus. If the cross-modal matching function is a power function of the form

$$
F=k N^{\epsilon} \text {, }
$$

and if the maxima of both continua $F_{\max }$ (the MVC) and $N_{\max }$ are perceived as equal by all subjects, ${ }^{1}$ then

$$
F_{\max }=k N_{\max }{ }^{\epsilon} \text {. }
$$

Under these circumstances, the exponent and scale of the cross-modal matching functions will covary with individual values of $F_{\max }$ : 


$$
\log k+\epsilon \log N_{\max }=\log F_{\max },
$$

provided that $N_{\max }$ is constant across subjects.

The second step requires that the form of the psychophysical function be constant and differ across subjects only by a scaling factor proportional to the maximum force. We can derive such an expression by dividing Equation 1 by Equation 2 to get

$$
F / F_{\max }=\left(N / N_{\max }\right)^{\epsilon} .
$$

If the exponent is constant across subjects, then equal fractions of the maximum force will be perceived equally. Furthermore, the scale constant will be proportional to the MVC. That is, from Equations 1 and 4,

$$
k=F_{\max } /\left(N_{\max }{ }^{\epsilon}\right) \text {. }
$$

The exponent, however, will be constant only if the dynamic range is constant across all subjects. If the minimum perceptible stimuli are $F_{\min }$ and $N_{\min }$, then from Equation 3,

$$
\begin{aligned}
\epsilon & =\left(\log F_{\max }-\log F_{\min }\right) /\left(\log N_{\max }-\log N_{\min }\right) \\
& =\left[\log \left(F_{\max } / \log F_{\min }\right)\right] /\left[\log \left(N_{\max } / N_{\min }\right)\right] .
\end{aligned}
$$

The exponent will be constant if the dynamic range $F_{\max } / F_{\min }$ is constant (assuming invariance of continuum $N$ across subjects). Equation 6 follows directly from Equation 2 of Teghtsoonian (1971), which he developed to explain differences in exponents of magnitude estimation functions across various continua. It serves here to predict exponent values across different subjects if their dynamic ranges are different. Rollman and Harris (1987) found that the exponents of individual magnitude estimation functions for the perceived pain of electrical shocks were correlated to the corresponding dynamic ranges for 40 subjects, consistent with Equation 6 . In contrast, Teghtsoonian, Teghtsoonian, and Karlsson (1981) failed to find the expected correlation between exponents of perceived exertion for cycling and the measured dynamic range across 30 subjects.

In sum, if maximal exertion is perceived equally by all subjects, as proposed by Borg $(1961,1962)$, then the exponent and scale of cross-modal matching functions between effort or exertion and an invariant reference continuum should covary with the maximum force. If the psychophysical function for perceived effort has the same form for all subjects, then the exponents should be constant and the scale factors should be proportional to the MVC. The purpose of this study is to use previously reported cross-modal matching data between pinch force, bite force, and an electrocutaneous stimulus (Van Doren et al., 1991) to test these predictions.

\section{METHOD}

\section{Subjects}

Six subjects participated in the cross-modal matching experiments: 3 males and 3 females, ages 24-32 years. All subjects were in good health, with no known sensorimotor problems.

\section{Electrocutaneous Stimuli}

The electrocutaneous stimuli were nearly identical to those first described by Saunders (1974) and those used subsequently by Sachs, Miller, and Grant (1980) in a cross-modal matching study of electrocutaneous stimulation and loudness. The stimuli consisted of bursts of pulses repeated at $30 \mathrm{~Hz}$ for $500 \mathrm{msec}$. The bursts contained between 1 and 255 constant-current $(7.5 \mathrm{~mA})$, biphasic pulses presented at $10 \mathrm{kHz}$. The cathodic phase of each biphasic pulse was presented first, followed by a $30-\mu \mathrm{sec}$ interphase interval and the anodic phase. The cathodic and anodic phases had equal durations ( 8 to $21 \mu \mathrm{sec}$ depending on subject), which were set $4 \mathrm{~dB}$ above the threshold for detecting a stimulus with a single pulse per burst (see below). The number of pulses in each burst was controlled by a microcomputer. The phase durations, pulse frequency, and burst frequency were controlled by a custom-built timer. The stimuli were generated using an isolated, constant-current stimulator. The stimuli evoked flutter-like sensations, characteristic of low-frequency mechanical vibrations, and the perceived intensity increased as the number of pulses increased.

The stimuli were delivered to the lateral surface of the upper arm, about halfway between the elbow and shoulder, via a concentric, bipolar, stainless steel electrode. The central, circular cathode had a diameter of $6.35 \mathrm{~mm}$, and the concentric, ring-shaped anode had an outer diameter of $25.4 \mathrm{~mm}$ and an inner diameter of $19.2 \mathrm{~mm}$. The anode and cathode were separated by a plastic insulator, recessed $1.6 \mathrm{~mm}$ from the electrode surface. The electrode was held in place using a strap, adjusted to be snug but not constrictive, and was consistently placed over a freckle or other skin landmark. Prior to placement, the skin was washed with alcohol and moistened with tapwater to stabilize the electrode impedance (Kaczmarek, Webster, Bach-y-Rita, \& Tompkins, 1991).

After placing the electrode on the subject, an approximate threshold was measured by adjusting the duration of the cathodic and anodic phases manually for a stimulus containing one pulse per burst until the subject reported just feeling the stimulus. The stimulus site was then conditioned (Saunders, 1974; Sachs et al., 1980) using a stimulus with 40 pulses per burst presented every $5 \mathrm{sec}$ for $2 \mathrm{~min}$. Following the conditioning period, the detection threshold was determined more carefully using the method of limits, taking the median of three ascending and three descending runs. The phase durations were then set at $4 \mathrm{~dB}$ SL for the remainder of the experiment. A second, 2-min conditioning interval followed the threshold measurements, with bursts containing $75 \%$ of the maximum number of pulses.

The number of pulses per burst used in the experiments depended on the tolerance of the subjects. Ten different burst durations (pulse numbers), logarithmically spaced, were used in each experimental session: 2-253 for 4 subjects, and 2-199 for 2 subjects. The sensation evoked by the 253-pulse stimulus, however, was qualitatively different from shorter bursts. It felt weaker and acquired a buzzing quality characteristic of the underlying $10-\mathrm{kHz}$ pulse repetition rate. The change was probably due to the long duty cycle of the stimulus $(25.5 \mathrm{msec}$ out of $33.3 \mathrm{msec})$. The matches made to burst durations of 253 pulses were not included in subsequent analyses.

\section{Grasp Force}

The subjects squeezed an instrumented cylinder between the thumb and first two fingers of the right hand in response to the electrocutaneous stimuli. The cylinder, shown in Figure 1, was $4.6 \mathrm{~cm}$ long, $2.5 \mathrm{~cm}$ wide at the bottom (thumb contact), and had a $3.3-\mathrm{cm}$ "cap" to contact the two fingers. The cap was connected to an inner piston that compressed a miniature load cell. The cylinder weighed $52 \mathrm{~g}$ and was held lightly by its sides with the left hand during the matching trials to avoid dropping the cylinder. Supporting the cylinder with the left hand allowed the subjects to apply very small pinch forces with the right hand, but it may have introduced slight differences in the force applied by the thumb and 


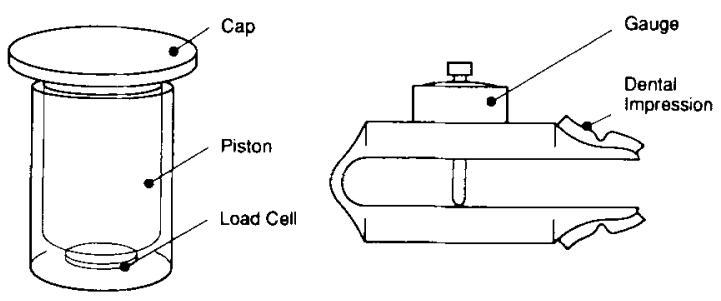

Figure 1. Sketches of force-measuring instruments. Left: Instrumented cylinder for measuring pinch force is held between the thumb (on bottom) and first two fingers (on top). The cap is connected to a piston that compresses a miniature load cell in the bottom of the cylinder. Right: Modified pinch meter for measuring bite force is held between the teeth, using custom dental impressions for each subject, and is partially supported by the subject's hand. The mechanical gauge has a peak-reading indicator. The pinch meter is enclosed in a clean plastic bag for each session.

the force applied by the two fingers. The output of the load cell was amplified, lowpass filtered at $10 \mathrm{~Hz}$, and sampled at $50 \mathrm{~Hz}$ using a 16-bit ADC (National Instruments, Austin, TX). The force was recorded during each trial, and an average force was then calculated off-line. The average force included only those points on a plateau within $80 \%$ of the peak value, and with a change in force less than $0.4 \mathrm{~N}$ between adjacent points (corresponding to a force rate of $20 \mathrm{~N} / \mathrm{sec}$ ). The use of both criteria rejected brief overshoots or transients, as well as data on the rising and falling edges of the force waveform (i.e., during squeeze and release).

\section{Bite Force}

Bite forces were measured using a mechanical pinch meter with a peak-reading force gauge (Figure 1). Dental impressions were made from thermoplastic for each subject and were mounted on the arms of the pinch meter. The meter was covered with a clean plastic bag for each session. The subject held the body of the meter lightly with one hand while biting the arms of the meter. The peakreading needle of the meter was reset to zero prior to each trial.

\section{Protocol}

Following the threshold measurements and conditioning periods in each session, cross-modal matches were made in five blocks of 10 trials. The 10 burst durations were presented in a random order within each block. The subject was given an auditory cue at the start of each trial, followed by the electrocutaneous stimulus. Two seconds after each stimulus, the subject was given a second auditory cue and was instructed to produce a brief pinch (or bite) force that had the same subjective magnitude as the sensation evoked by the electrocutaneous stimulus. There was a 5-sec pause between trials to reduce the effects of fatigue and adaptation. The maximum pinch force was measured at the beginning and end of each session. Maximum bite force was not measured, to avoid possible dental or periodontal injury.

All sessions were on separate days, and bite force sessions followed the pinch force sessions. Five subjects completed four pinch force sessions, the 6th subject (C.V.D.) completed six sessions, and all subjects completed four bite force sessions.

\section{RESULTS AND DISCUSSION}

The cross-modal matching functions for the 6 subjects are shown in Figure 2. The data from each session was reduced by taking the median force across five trials for each burst duration. Each point in Figure 2 represents the geometric mean of the medians from four sessions (or six sessions for Subject C.V.D., pinch). The lines are the best-fitting power functions to the session medians.

The power function parameters and the MVCs for each subject are listed in Table 1. The scale factors of the power functions $(\log k)$ for both pinch and bite are negatively correlated with their exponents $(\epsilon)$, as shown in Figure 3. The filled points in Figure 3 represent the parameters for Subject D.E.W., who had a very steep pinch-force matching function with a low intercept that was not consistent with the overall performance of the other 5 subjects.

Apart from Subject D.E.W., there was a strong positive correlation between the MVCs and the scale factors of the pinch functions, and a negative correlation between the MVCs and the exponents. A one-way analysis of variance (ANOVA) showed that the variation in both parameters (excluding Subject D.E.W.) was significant $[\log k$, $F(4,191)=62.5, p<.01 ; \epsilon, F(4,191)=3.80, p<$ $.01]$. Games-Howell pairwise comparisons (Games, Keselman, \& Rogan, 1981) showed that the exponents of Subjects J.C.B. and M.L.H. were significantly different from the exponent of Subject K.L.M. $(p<.05)$ and
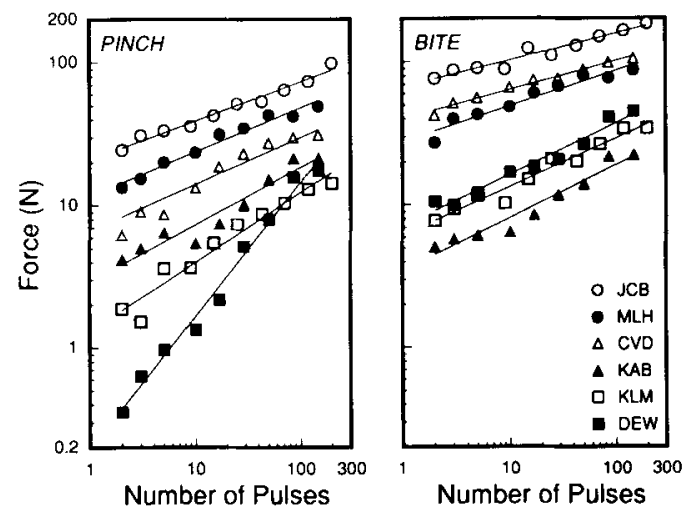

Figure 2. Cross-modal matching functions between pinch force (left) or bite force (right) and an electrocutaneous stimulus. The intensity of the electrocutaneous stimulus was controlled by the number of pulses in each burst of the stimulus. Five matches were made for each of 10 pulse numbers in each session. The lines are bestfitting power functions to the medians from each of four sessions (except for Subject C.V.D., who completed six pinch force sessions), and the points are the geometric means of the session medians. Note that Subject D.E.W. (filled squares) had an unusually steep pinch force function.

Table 1

Maximum Pinch Forces, and Exponents $(\epsilon)$ and Scale Factors $(\log k)$ for Pinch and Bite Cross-Modal Matching Functions

\begin{tabular}{|c|c|c|c|c|c|}
\hline \multirow[b]{2}{*}{ Subject } & \multirow[b]{2}{*}{ Max Pinch (N) } & \multicolumn{2}{|c|}{ Grasp } & \multicolumn{2}{|c|}{ Bite } \\
\hline & & $\epsilon$ & $\log k$ & $\epsilon$ & $\log k$ \\
\hline$\overline{\text { K.L.M. }}$ & 68.9 & 0.48 & 0.12 & 0.34 & 0.79 \\
\hline K.A.B. & 76.7 & 0.40 & 0.47 & 0.37 & 0.54 \\
\hline C.V.D. & 121.1 & 0.33 & 0.82 & 0.20 & 1.60 \\
\hline M.L.H. & 121.3 & 0.31 & 1.07 & 0.25 & 1.44 \\
\hline J.C.B. & 164.8 & 0.27 & 1.32 & 0.19 & 1.83 \\
\hline D.E.W. & 74.0 & 0.93 & -0.71 & 0.36 & 0.85 \\
\hline
\end{tabular}



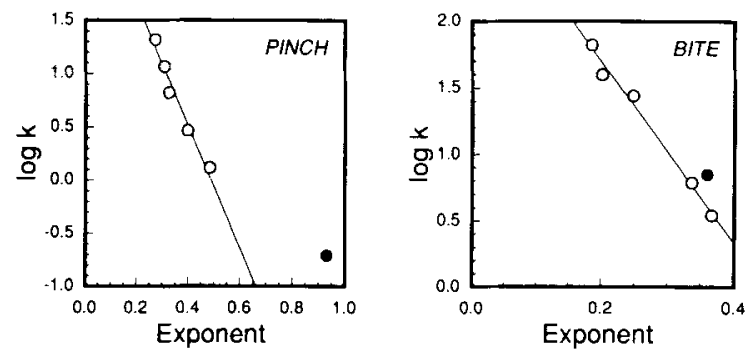

Figure 3. Exponents of the best-fitting power functions plotted versus the scaling factors $(\log k)$ for pinch (left) and bite (right). The filled points represent the parameters for Subject D.E.W. The exponents for the other subjects are linearly related to the scaling factors, as indicated by the best-fitting lines.

all of the estimates of the intercept were different from each other. A one-way ANOVA of the logarithm of the MVC (data pooled across all trials and sessions ${ }^{2}$ ) showed that the overall variation was significant across subjects $[F(4,38)=109.2, p<.0001]$. All pairwise comparisons were significantly different $(p<.05)$ except those for Subject M.L.H. versus Subject C.V.D. and Subject K.L.M. versus Subject K.A.B.

Scale factors and exponents also varied significantly across subjects $(p<.01)$ for bite force matching. Pairwise comparisons showed that the exponents of Subjects J.C.B. and C.V.D. were significantly different from those of Subjects K.L.M. and K.A.B., and all of the scale factors were different from each other.

Given that the exponents, scale factors, and maximum pinch forces varied significantly across subjects, we can reject the simplest hypotheses that pinch force matches are made independent of strength and that matching function slopes are constant. We can then ask whether these variations can be predicted from the theory of RPE. If the first component of the theory is correct-that is, that the sensation at the maximum force is constant across all subjects-and if we assume a constancy for the maximum number of pulses, then the measured MVC should be a function of the exponent and scale factor according to Equation 3.

From the pinch parameters and MVCs in Table 1, an iterative procedure was used to select a value of $N_{\max }=$ 3,478 , which minimized the mean squared difference between the predicted and measured MVCs, excluding Subject D.E.W. ${ }^{3}$ The predicted values are shown in Figure 4 (open points) and are in good agreement with the actual MVCs $\left(r^{2}=0.86, \log\right.$ - $\log$ correlation, $\left.p<.05\right)$. The filled point is the predicted MVC calculated using the same value of $N_{\max }$ for Subject D.E.W. Clearly, the postulate that pinch maxima are perceived equally does not account for Subject D.E.W., but it does provide a good fit for the other 5 subjects.

The theory is also supported indirectly by the bite force cross-modal matching functions. The parameters of the bite functions (Table 1) appear to be correlated with the maximum pinch forces. Although maximum bite forces could not be measured safely, theoretical MVCs were calculated using Equation 3 and the same value of $N_{\max }$ derived from the pinch force functions. The predicted bite MVCs are plotted as a function of the measured pinch MVCs in Figure 4. The values are strongly correlated $\left(r^{2}=0.91, p<.05\right.$; excluding Subject D.E.W., filled point), suggesting that bite forces are generated relative to a subject's maximum bite force, provided that the maximum bite force is correlated to maximum pinch force (perhaps by virtue of a global relationship between body size and strength).

The second component of the theory-that is, that the perceived exertion was constant across subjects at any fixed fraction of the maximum level-is not supported by either the pinch or bite data. If it were true, then all of the cross-modal matching functions would have the same exponent. As mentioned above, the exponent varied significantly across subjects for both bite force and pinch force. The variation in the exponent suggests that the dynamic range $F_{\max } / F_{\min }$ is not constant across subjects, according to Equation 6. The "minimum exertable force" was not measured, though, so we cannot compare the inferred variation in dynamic range with an objective measure.

The matching data are parsimonious with the condition that subjects experience the same perceived effort at their respective maximal pinch forces (and perhaps for bite forces as well), provided that the electrocutaneous continuum is invariant across subjects. It is important to recognize, however, that this condition alone does not predict the observed correlation of either the scale factors or exponents with the MVCs. Equation 3 only predicts a correlation between a particular combination of the two parameters and the maximum force. For example, Subject D.E.W. had the lowest scale factor and the highest exponent of the 6 subjects. The trends displayed by the other
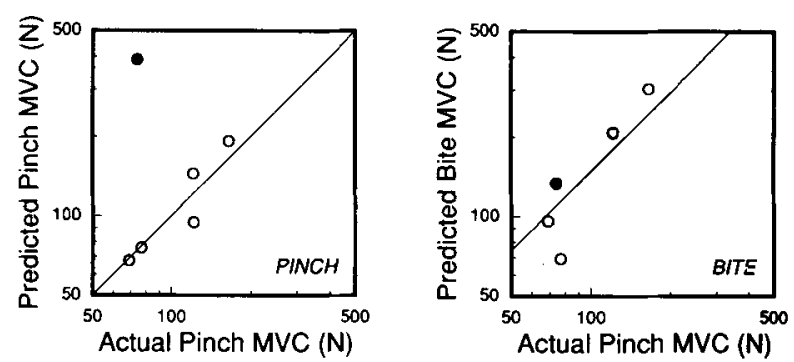

Figure 4. Left: Maximum pinch forces (MVCs) predicted using the exponents and scale factors for each subject, plotted versus the measured MVCs. An iterative procedure was used to optimize the fit (Equation 3) for 5 subjects (open points). The filled point is the MVC predicted for D.E.W. based on the relation determined for the other 5 subjects. The line represents equality between the predicted and measured MVCs. Right: Maximum bite forces, predicted from the relation derived for pinch force, plotted as a function of the actual pinch MVC. The line represents proportionality between the predicted bite MVC and the measured grasp MVC. 
subjects would indicate that her MVC should be small, yet her function would fit the predictions of Equation 3 if her MVC had actually been quite high, about $400 \mathrm{~N}$ (Figure 3).

There may be a second, rather curious, condition that can account for the correlations between $\epsilon$ and $\log k$ and the MVC. The linear relationship between the exponents and scale factors is nearly identical for both pinch force and bite force, and all of the parameters (excluding Subject D.E.W.) are plotted together in Figure 5. In fact, the slopes and intercepts of the grasp and bite parameter plots in Figure 3 are not significantly different $(p>.10)$. A linear relationship between the slopes and intercepts for any family of lines implies that all of the lines converge at a common point (Stevens, 1974). The linear relationship between exponents and scale factors, then, implies that all of the matching functions intersect at a point. That is, all the subjects perceive a single absolute force (rather than a relative force) equally, for both bite and pinch, and they equate it to the same burst duration. From the slope $m$ and intercept $b$ of the line in Figure 5, the coordinates of the point of convergence $\left(N_{c}, F_{c}\right)$ are given by

$$
\begin{aligned}
& \log N_{c}=-m \\
& \log F_{c}=b,
\end{aligned}
$$

which gives $F_{c}=713 \mathrm{~N}$ (95\% confidence interval, 430$1,181 \mathrm{~N})$, and $N_{c}=8.49 \times 10^{5}$ pulses per burst $(95 \%$ confidence interval, $1.79 \times 10^{5}$ to $27.2 \times 10^{5}$ pulses).

The two conditions supported by the data are represented schematically in Figure 6. The lines represent individual matching functions for either bite force or pinch force. Each line passes through the subject's MVC at a burst duration of $N_{\max }$, and all of the lines pass through the point $\left(N_{c}, F_{c}\right)$. The slopes and intercepts of the functions are negatively correlated: the slopes decrease with increasing MVC $\left(F_{\max }\right)$, and the intercepts increase with increasing MVC. The data cannot reject this model on the basis of lack of fit, but the values necessary for convergence seem impossibly high, and there is no obvious

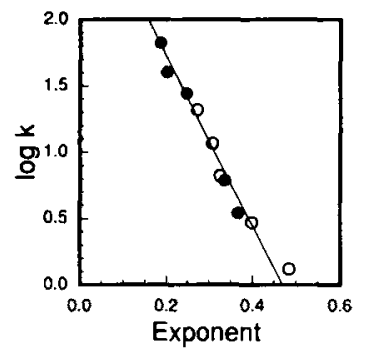

Figure 5. Exponents plotted versus scale factors for both pinch (open points) and bite (filled points), excluding the parameters of Subject D.E.W. Both sets of parameters are fit well by a single line, which indicates that all of the matching functions converge at a single point. The coordinates of the convergence point are $F_{c}=713 \mathrm{~N}$, $N_{c}=8.49 \times 10^{s}$, as calculated from the slope and intercept of the line.

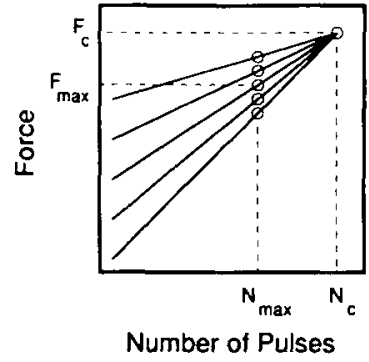

Figure 6. Idealized drawing of force perception model. Each line represents an individual pinch force or bite force cross-modal matching function. All of the functions converge at a single point $\left(N_{c}, F_{c}\right)$, and each line passes through the subject's MVC (i.e., $F_{\max }$ ) at a single value of $\boldsymbol{N}_{\max }$.

reason for both pinch and bite functions to converge. In several previous studies, families of magnitude estimation functions have converged at single points, but always for functions averaged across individuals tested under different conditions. Thirteen such families were summarized by Stevens (1974) including perceived brightness, auditory volume, heaviness, warmth, and cold. Stevens and Marks (1971; see also Marks \& Stevens, 1973), for example, obtained magnitude estimation functions for perceived warmth versus irradiance on the forehead, and varied the irradiated area parametrically. The functions get steeper as the area decreases; when extrapolated, they converge at a single point (numerical estimate of about 55 , irradiance of $800 \mathrm{~mW} / \mathrm{cm}^{2}$ ). In this case, the point of convergence corresponds roughly to the threshold of pain, which is nearly independent of stimulus area (e.g., Greene \& Hardy, 1958). Magnitude estimation functions for perceived heaviness of lifted weights display a similar convergence to a point corresponding to the maximum heft (Stevens \& Rubin, 1970). Finally, Stevens (1990) has found that perceived roughness functions for grooved plates applied to different body loci converge at a common, minimal value (a groove width of $0.13 \mathrm{~mm}$ ), possibly corresponding to a stimulus that feels smooth everywhere on the body.

The convergence reported here does not have an intuitively plausible explanation. The functions converge across different subjects, implying a constant perceived value of some absolute stimulus level, and the force at the point of convergence $(713 \mathrm{~N})$ is beyond the physical capacity of the system, at least for pinch. We do not know whether subjects could attain a bite force of $713 \mathrm{~N}$, nor do we know if a subject could tolerate an electrocutaneous stimulus with an intensity equivalent to a burst with 850,000 pulses. Further experiments are needed, covering a wider range of forces and including more subjects with different strengths, to see if the convergence is robust and to determine whether it occurs for different reference continua and different muscle groups. 


\section{CONCLUSIONS}

Cross-modal matches of both pinch force and bite force were power functions of the burst duration (i.e., the number of pulses per burst) in an electrocutaneous stimulus. The exponents and scale factors for the pinch-matching functions covaried with the maximum pinch force and were consistent with the condition that all subjects perceived their physical maxima equally, in support of Borg's theory of relative perceived exertion. However, since the exponents varied significantly across subjects, forces at any given fraction of the maximal forces were not perceived equally. Finally, the exponents and scale factors for both bite and pinch were linearly related, which suggests that all of the matching functions converged at a single point perceived equally by all subjects, corresponding to a very high absolute force and burst duration.

\section{REFERENCES}

BoRG, G. (1961). Interindividual scaling and perception of muscular force. Kungliga Fysiografiska Sällskapets i Lund Fördhandlingar, 31, 117-125.

BORG, G. (1962). Physical performance and perceived exertion. Lund: Gleerup.

Cafarelli, E., \& Bigland-Rutchie, B. (1979). Sensation of static force in muscles of different length. Experimental Neurology, 65, 511-525.

Games, P. A., Keselman, H. J., \& Rogan, J. C. (1981). Simultaneous pairwise multiple comparison procedures when sample sizes are unequal. Psychological Bulletin, 90, 594-598.

GREENE, L. C., \& HARDY, J. D. (1958). Spatial summation of pain. Journal of Applied Physiology, 13, 457-464.

Kaczmarek, K. A., Webster, J. G., Bach-y-Rita, P., Tompkins, W. J. (1991). Electrotactile and vibrotactile displays for sensory substitution. IEEE Transactions on Biomedical Engineering, 38, 1-16.

Marks, L. E., Borg, G., \& LuUNGgren, G. (1983). Individual differences in perceived exertion assessed by two new methods. Perception \& Psychophysics, 34, 280-288.

MARKs, L. E. \& STEVENS, J. C. (1973). Spatial summation of warmth: Influence of duration and configuration of the stimulus. American Journal of Psychology, 86, 251-267.

McCloskey, D. I., Ebeling, P., \& Goodwin, G. M. (1974). Estimation of weights and tensions and apparent involvement of a "sense of effort." Experimental Neurology, 42, 220-232.

Minevic, P. M. (1983). Cardiovascular fitness and the psychophysics of perceived exertion. Research Quarterly for Exercise \& Sport, 54, 239-246.

Morgan, W, P., Pollock, M. L. (1977). Psychologic characterization of the elite distance runner. Annals of the New York Academy of Science, 301, 382-403.

Nagle, F. J., Morgan, W. P., Hellickson, R. O., Serfass, R. C., \& Alexander, J. F. (1975). Spotting success traits in Olympic contenders. Physician \& Sports Medicine, 12, 31-34.
Patton, J. F., Morgan, W. P., \& Vogel, J. A. (1977). Perceived exertion of absolute work during a military training program. European Journal of Applied Physiology, 36, 107-114.

Redline, S., Gottfried, S. B., Altose, M. D. (1991). Effects of changes in inspiratory muscle strength on the sensation of respiratory force. Journal of Applied Physiology, 70, 240-245.

Roland, P. E., \& LADEgaARd-Pedersen, H. (1977). A quantitative analysis of sensations of tension and kinaesthesia in man. Brain, $\mathbf{1 0 0}$ 671-692.

Rollman, G. B., \& Harris, G. (1987). The detectability, discriminability, and perceived magnitude of painful electrical shock. Perception \& Psychophysics, 42, 257-268.

Sachs, R. M., Miller, J. D., \& Grant, K. W. (1980). Perceived mag nitude of multiple electrocutaneous pulses. Perception \& Psychophysics, 28, 255-262.

SAUNDERS, F. A. (1974). Electrocutaneous displays. In F. A. Geldard (Ed.), Cutaneous communication systems and devices (pp. 20-26) Austin, TX: Psychonomic Society.

Stevens, J. C. (1974). Families of converging power functions in psychophysics. In H. R. Moskowitz \& B. Scharf (Eds.), Sensation and measurement (pp. 157-165). Dordrecht-Holland: D. Reidel.

Stevens, J. C. (1990). Perceived roughness as a function of body loci. Perception \& Psychophysics, 47, 298-304.

Stevens, J. C., \& CaIN, W. S. (1970). Effort in isometric muscular contractions related to force level and duration. Perception \& Psycho physics, 8, 240-244.

Stevens, J. C., \& Marks, L. E. (1971). Spatial summation and the dynamics of warmth. Perception \& Psychophysics, 9, 391-398.

Stevens, J. C., \& Rubin, L. L. (1970). Psychophysical scales of apparent heaviness and the size-weight illusion. Perception \& Psychophysics, 8, 225-230.

Teghtsoonian, R. (1971). On the exponent in Stevens' law, and the constant in Ekman's law. Psychological Review, 78, 71-80.

Teghtsoonian, R., Teghtsoontan, M., \& KarLsSon, J.-G. (1981) The limits of perceived magnitude: Comparison among individuals and among perceptual continua. Acta Psychologica, 49, 83-94.

Van Doren, C. L., Riso, R. R., \& Milchus, K. L. (1991). Sensory feedback for enhancing upper extremity neuromuscular prostheses. Journal of Neurological Rehabilitation, 5, 63-74.

\section{NOTES}

1. It is not actually necessary that $N_{\max }$ be the maximum of the $N$ continuum; it only represents that value of $N$ that generates the same sensation as $F_{\max }$. The subsequent expressions that are derived assume a convenient symmetry, however, using this notation.

2. A two-way ANOVA of the MVCs pooled across subjects showed no significant effect of session or trial (i.e., beginning or end of session).

3. Note that this number of pulses cannot be physically accommodated in our stimulus pattern with bursts repeated at $30 \mathrm{~Hz}$. This number simply corresponds to a sensation that cannot be evoked with our particular stimulus pattern.

(Manuscript received January 31, 1992; revision accepted for publication October 20,1992 .) 